\title{
Be Binaries with Warm Dust and Exotic High-Luminosity A-F Emission-Line Stars
}

\author{
Anatoly S. Miroshnichenko \& Karen S. Bjorkman \\ Ritter Observatory, Dept. of Physics \& Astronomy, University of \\ Toledo, Toledo, OH 43606, USA \\ Valentina G. Klochkova \& Eugene L. Chentsov \\ Special Astrophysical Observatory of the Russian Academy of Sciences, \\ Karachai-Chircassian Republic, Nizhnij Arkhyz, 369167, Russia
}

\begin{abstract}
We present the results of photometric and high-resolution spectroscopic studies of a group of $\mathrm{B}[\mathrm{e}]$ stars (Be stars with warm dust or $\mathrm{B}[\mathrm{e}] \mathrm{Ds})$ and of two cooler objects with extremely high luminosities (HDE 331319 and IRC+10420). The $\mathrm{B}[\mathrm{e}] \mathrm{Ds}$ is a growing group of objects with very strong emission-line spectra, some of which show direct evidence of the presence of a hot and a cool star in the system. Their basic properties and connection to other groups of binaries are discussed, and a new list of members is presented. Fundamental parameters and chemical composition of an unusual F-supergiant HDE 331319 and recent results on a fast evolving object IRC +10420 are also reported.
\end{abstract}

\section{Introduction}

Formation of circumstellar (CS) dust in systems containing evolved early-type stars requires special conditions and is always an interesting subject for a study. In this talk our new results on three different types of such objects are presented.

\section{Be stars with warm dust}

$\mathrm{B}[\mathrm{e}]$ stars are emission-line B-type stars showing emission-line spectra and strong near-IR excesses. This group containing 65 galactic objects was first selected by Allen \& Swings (1976), who studied $\sim 700$ early-type emission-line stars and noticed a correlation between the appearance of forbidden emission lines and the near-IR excess due to CS dust. The original list of $B[e]$ stars turned out to be rather heterogeneous in terms of the nature and evolutionary state of the members. Such objects as Herbig Ae/Be stars, supergiants and LBVs, symbiotic systems, PPNe and PNe were recognized there. However, it turned out that 28 out of the 65 objects still have an unknown nature (unclassified B[e] stars, see Lamers et al. 1998). The group can also be enlarged from the lists of Carlson \& Henize (1979) and Dong \& Hu (1991).

CS dust can easily be formed around evolved cool stars, which produce heavy elements in their interiors and transport them out of the atmospheres. At 
the same time, many hot stars with evidence for CS dust are known, and it is relevant here to review separate groups of such objects including binary systems with a hot companion. In pre-main-sequence and main-sequence intermediatemass stars (Herbig Ae/Be and Vega-type stars, respectively), the dust has a protostellar origin. It was formed by previous generations of stars and takes part in comet and possibly planet formation around these young systems. In symbiotic and VV Cep-type binaries, M-type companions seem to be responsible for this process. In Wolf-Rayet stars, special conditions exist for dust formation (the presence of carbon without hydrogen, high matter densities, and colliding winds). In LBVs, the dust is probably formed during their violent eruptions (which may involve high densities), then it is swept out by the powerful winds, and therefore cold. Planetary $(\mathrm{PN})$ and proto-planetary nebulae (PPN) have dust formed at the previous, AGB, phase of their evolution. Only in B[e] stars, where the dust is certainly hot and located close to the star, is the origin of its formation process not understood yet.

Our interest in $\mathrm{B}[\mathrm{e}]$ stars (mainly in the unclassified members) began some 15 years ago, with an attempt to identify new Herbig Ae/Be stars. This project gradually evolved into a study of what we tentatively call Be stars with warm dust (B[e]Ds). All B[e] stars were detected by the IRAS satellite, and we noticed that a large group of $\mathrm{B}[\mathrm{e}]$ and similar objects, associated with hot emission-line stars, have specific IRAS colors $\left(-0.5 \leq \log F_{12} / F_{25} \leq 0.1,-1.1 \leq \log F_{60} / F_{25} \leq\right.$ $-0.3)$ which correspond to dust temperatures of $\geq 150-200 \mathrm{~K}$. Such colors are characteristic of mainly late-type stars with CS dust (symbiotic and VV Cep binaries, Miras) and may indicate either the presence of a cool companion or a compact dusty envelope (lack of cold dust). The latter may be due to a recent dust formation process. It is unlikely that the $\mathrm{B}[\mathrm{e}] \mathrm{Ds}$ are young stars because of no association with star forming regions, lack of far-IR excess with respect to the near-IR one, and the high luminosities of some of the objects (too massive to have an observable pre-main-sequence stage). In total we found 19 galactic objects with the mentioned color-indices. Some of their parameters are presented in Table 1. Our first report on this group was published by Sheikina, Miroshnichenko, \& Corporon (2000). Since that time 8 new objects have been added to the group, and many new observations have been obtained. It should be emphasized here that these objects have been poorly investigated until recently because of a lack of systematic observations (especially high-resolution spectroscopy), interferometric data, and coordinated programs. Some results of our efforts to organize an observational campaign and advance in understanding of B[e]Ds were summarized in Miroshnichenko et al. (2000, 2001, 2002).

The main observational properties of $B[e] D s$ can be summarized as follows: optical colors of reddened early-type stars; emission-line spectrum dominated by strong Balmer lines and numerous Fe II and [Fe II] lines; strong lines of [O I] are usually present. Only a few objects display photospheric lines of B-type stars, while most of the others do not show any photospheric lines at all. The luminosities and initial masses seem to be very different (see Table 1); the line profile shapes indicate a disk-like geometry of the CS gas, which may also be the case for the CS dust.

There are a few systems with evidence for the secondary component. In MWC 623, HDE 327083 and AS 381 photospheric lines of the cool component 
are clearly seen. In AS 381 and V669 Cep molecular bands of CN and CO are observed in the near-IR. In all these cases, the secondaries were suggested to be K-type giants (no molecular bands are seen in the optical spectra) or bright giants. CI Cam became famous after an all-wavelength eruption on $1998 \mathrm{March}$ 31 , including $\gamma$ - and X-ray outbursts which suggested the presence of a compact object (a neutron star or a black hole) as secondary. We also suspected that a few other B[e]Ds (e.g. MWC 342, MWC 657, Hen 1398) might be binaries, because of their cyclic brightness variations and unusual spectral line profiles. No information about orbital parameters is known yet.

Consider some of the mentioned properties of $\mathrm{B}[\mathrm{e}] \mathrm{Ds}$. The Balmer line emission is extremely strong in most cases (see Table 1). Such a strong emission may be due to either very high mass-loss rates or high densities in the envelope. The line profiles are usually double- or single-peaked, indicating a disk-like gas distribution, and they are rarely of P Cyg-type, which is more consistent with spherical geometry. There is a correlation between the $\mathrm{H} \alpha$ line EW and orbital period for Be/X-ray binaries (Reig, Fabregat, \& Coe 1997) which seems to hold for classical Be binaries. It can be explained by the fact that Roche lobes are larger and are capable of storing more material in wider binaries. In the case of binary $\mathrm{B}[\mathrm{e}] \mathrm{Ds}$, this might mean that the orbital periods are even larger than in Be stars (up to a few years). Furthermore, larger distances from hot stars inside their Roche lobes may allow dust formation, just because the temperature can be low enough for that. On the other hand, the dust may exist around the cool companion or around the whole binary.

The ISO spectra of $\mathrm{B}[\mathrm{e}] \mathrm{Ds}$ show the presence of both oxygen- and carbonrich dust. The silicate crystallinity indicates that the dust has been processed for a long time, favoring an evolved evolutionary state (Voors 1999). The IRAS low-resolution spectra are featureless or show silicate features in emission. The former may be an optical depth effect, or may be due to dust composed of amorphous carbon. An optically thick dust, if formed around the cool companion, may produce an additional obscuration of the star and explain why the hot companions are brighter in AS 381, V669 Cep, and possibly HDE 327083 . Only in MWC 623 , the K-type star seems to be visually brighter than the B-type star (Zickgraf 2001).

All B[e]Ds that have been extensively observed photometrically show significant brightness variations on timescales from days to years. A typical amplitude varies from 0.3 to $0.8 \mathrm{mag}$ with an exception of CI Cam, which brightened by $\sim 3$ mag during its 1998 outburst.

A few $B[\mathrm{e}] \mathrm{Ds}$ were considered to be supergiants. This suggestion was based on such indirect properties as the presence of Fe III emission lines, which seem to be observed only in hypergiants (MWC 300, Wolf \& Stahl 1985), or extremely strong interstellar Na I D-lines (HDE $327083, \mathrm{CPD}-57^{\circ} 2874$, and CPD $-52^{\circ} 9243$, Lopes, Damineli Neto, \& de Freitas Pacheco 1992). However, their luminosities still have to be verified. For example, the radial velocity of MWC 300 suggests a distance of $\sim 2 \mathrm{kpc}$, which significantly reduces its luminosity. With such a luminosity it is still a supergiant, but not a hypergiant at the Eddington limit.

Summarizing, we suggest that B[e]Ds form a distinct group of binaries (at least most of them). Binarity seems to be responsible for the major observational 
properties of these objects, such as the emission-line strengths and IR colors. The $\mathrm{B}[\mathrm{e}] \mathrm{Ds}$ are probably less evolved than symbiotic and VV Cep stars (mainly $\mathrm{K}$-type secondaries and non-degenerate hot companions). However, a number of questions need to be further addressed on the way to a better understanding of the $\mathrm{B}[\mathrm{e}] \mathrm{D}$ physics. They include a search for traces of the secondaries in more $\mathrm{B}[\mathrm{e}] \mathrm{Ds}$; the problem of the companions' luminosity ratio (why cool companions are fainter); geometry of both gaseous and dusty envelopes. Regular observations are needed to constrain the orbital periods. High-resolution mid-IR observations are important to resolve the dusty envelopes of $\mathrm{B}[\mathrm{e}] \mathrm{Ds}$ and thus put constrains on the dust location within the systems.

About 10000 IRAS sources have IR colors similar to those of B[e]Ds. Nearly 2000 of them can be identified with late-type optical counterparts from the HD catalog. The other $\sim 8000$ seem to be fainter, and a separate study has to be done to identify these objects. Our quick look resulted in 2 new B[e]D candidates, Hen 298 and Hen 303 , in which we have already detected strong IR excesses. This suggests that the group can be further enlarged.

Table 1. Be stars with warm dust

\begin{tabular}{|c|c|c|c|c|c|c|c|}
\hline Name & IRAS & $\bar{V}$ & Sp.T. & $\bar{D}$ & $\log L / L_{\odot}$ & $E_{B-V}^{t o t}$ & $\mathrm{H} \alpha$ \\
\hline AS 78 & $03549+5602$ & $11.2-11.4$ & B2/4 & 2.5 & $3.9 \pm 0.1$ & 0.90 & 115 \\
\hline CI Cam & $04156+5552$ & $9.0-11.6$ & $\mathrm{~B} 0 / 2+?$ & 1.5 & $5.0 \pm 0.5$ & 1.10 & $250^{\mathrm{a}}$ \\
\hline HD 45677 & $06259-1301$ & $6.9-8.8$ & B2 & 0.5 & $3.5 \pm 0.4$ & 0.20 & 170 \\
\hline HD 50138 & $06491-0654$ & $6.5-6.8$ & B5 $/ 8$ & 0.3 & $2.9 \pm 0.2$ & 0.15 & 60 \\
\hline AS 160 & $07370-2438$ & 10.9 & $\mathrm{~B} 1$ & & $4.0 \pm 0.1$ & & 250 \\
\hline Hen 140 & $08128-5000$ & 10.1 & $\mathrm{~B} 2 / 8$ & 2.0 & $3.1 \pm 0.2$ & 0.30 & \\
\hline Hen 298 & $09350-5314$ & 11.4: & B & & & & 330 \\
\hline Hen 303 & $09369-5406$ & 13.1: & B & & & & \\
\hline HD 85567 & $09489-6044$ & 8.6 & $\mathrm{~B} 2$ & 1.5 & $4.0 \pm 0.3$ & 0.40 & 31 \\
\hline$-57^{\circ} 2874$ & $10136-5736$ & 10.1 & $\mathrm{~B} 3 / 5$ & 2.5 & & 1.85 & \\
\hline$-52^{\circ} 9243$ & $16031-5255$ & 10.3 & $\mathrm{~B} 3 / 4$ & 4.9 & $5.7 \pm 0.3$ & 1.75 & $57-83$ \\
\hline HDE 327083 & $17117-4016$ & 9.7 & $\mathrm{~B} 1 / 2+\mathrm{K}$ & 5.0 & 6.3 & 1.80 & 36 \\
\hline Hen 1398 & $17213-3841$ & 10.6 & O9 & 3.3 & $5.3 \pm 0.2$ & 1.10 & \\
\hline MWC 300 & $18267-0606$ & $11.5-11.7$ & B1 & 15 & 5.7 & 1.20 & $140 \pm 10$ \\
\hline MWC 623 & $19545+3058$ & $10.5-10.9$ & $\mathrm{~B} 2+\mathrm{K} 2$ & 2.4 & $3.7 \pm 0.4$ & 1.40 & $122 \pm 5$ \\
\hline AS 381 & $20047+3305$ & 14.4 & $\mathrm{~B} 1+\mathrm{K}$ & 4.0 & $4.9 \pm 0.2$ & 2.20 & $\geq 85$ \\
\hline MWC 342 & $20212+3920$ & $10.2-10.9$ & $\mathrm{~B} 1 / 2$ & 1.0 & $4.1 \pm 0.4$ & 1.40 & $\overline{200} \pm 15$ \\
\hline V669 Cep & $22248+6058$ & $12.0-12.4$ & $\mathrm{~B} 5+\mathrm{K}$ & 1.0 & $2.7 \pm 0.3$ & 0.90 & $67-186$ \\
\hline MWC 657 & $22407+6008$ & $12.3-12.9$ & B1 & 2.0 & $3.7 \pm 0.3$ & 1.60 & 180 \\
\hline
\end{tabular}

$D$ is the distance from the Sun in kpc (column 5); $E_{B-V}^{t o t}$ (column 7) is the overall reddening determined from the observed and intrinsic color-indices; the equivalent width of the $\mathrm{H} \alpha$ line is given in column 10.

Measured during the quiescent state

\section{HD 331319}

HD $331319=$ IRAS $19475+3119$ is an F-type high-luminosity star and a PPN candidate, first identified in the IRAS survey. It shows the presence of cold dust in the far-IR region, a low optical reddening $\left(\mathrm{E}_{B-V} \sim 0.3 \mathrm{mag}\right.$ ), and almost no near-IR excess. A few near-IR observations, obtained in 1990-2000, show small brightness variations of $\sim 0.05$ mag. Detection of a weak CO $(2 \rightarrow 1)$ emission was reported by Likkel et al. (1991), while no maser emission in CS, 
$\mathrm{H}_{2} \mathrm{O}$ or $\mathrm{OH}$ was detected. The near- and mid-IR emission bands (due to PAHs or carbonaceous species) are not present in its spectrum. The kinematical distance is $\sim 6 \mathrm{kpc}$. Imaging polarimetry shows that the object has a two-armed spiral structure, probably due the interaction of the mass-losing star with a binary companion (Gledhill et al. 2001).

High-resolution spectra of HD 331319 were obtained at the 6-meter telescope of the Russian Academy of Sciences with the échelle-spectrograph PFES $(R \sim 15000$; 4 observations in July 1997 and April-June 2000; spectral range 4300-8000 $\AA$ ). The results, published in Klochkova et al. (2002), can be briefly summarized as follows.

A strong IR O I triplet ( $\mathrm{EW}=2.6 \AA$ ), corresponding to an $\mathrm{M}_{V} \leq-8 \mathrm{mag}$, was detected. The $\mathrm{H} \alpha$ line has a variable double-peaked profile with a deep central depression, which is probably due to a disk-like CS geometry. Using the model atmosphere method, the following parameters were determined: $T_{\text {eff }}=$ $7200 \mathrm{~K}, \log g=0.5,[\mathrm{~N} / \mathrm{Fe}]=1.30 \mathrm{dex},[\mathrm{O} / \mathrm{Fe}]=0.64 \mathrm{dex}, \mathrm{O} / \mathrm{C} \geq 1$. No radial velocity variations have been found. Weak He I lines at 4471, 4713, 4921, 5876, and $7065 \AA$, suggesting an enhanced $\mathrm{He}$ abundance of $[\mathrm{He} / \mathrm{Fe}]=1.63 \mathrm{dex}$, are present.

Thus, HD 331319 may be one of the most luminous PPN candidates with a strongly enhanced He abundance belonging to the galactic disk population. There is an apparent contradiction between the estimated distance $(6 \mathrm{kpc})$ and a small color-excess $\left(\mathrm{E}_{B-V} \sim 0.4 \mathrm{mag}\right)$, given the low galactic latitude $(b=2.7)$. According to Neckel \& Klare (1980), an $\mathrm{E}_{B-V}$ of $\sim 0.8 \mathrm{mag}$ is observed in this direction at only $2-3 \mathrm{kpc}$.

\section{IRC +10420}

IRC +10420 is an undoubtedly exotic object. In a recent paper (Klochkova et al. 2002), 3 spectra of this star obtained in May 1997, July 1999, and April 2000 (échelle-spectrometer PFES, $R=15000$, spectral range 4300-8000 $\AA$ ) were compared. The main results are as follows. The 2000 spectrum of IRC+10420 is very similar to that of $\alpha$ Cygni (A2 Ia) suggesting a $T_{\text {eff }} \sim 9200 \mathrm{~K}$. The same conclusion was reached by Oudmaijer (1998). Thus, for the 27 years since the first spectral classification of the star, its temperature increased by more than $3000 \mathrm{~K}$ or $\sim 120 \mathrm{~K}$ a year. The published distance $(3.4-6.8 \mathrm{kpc})$ and interstellar extinction estimates $\left(\mathrm{A}_{V}=6-7 \mathrm{mag}\right)$ suggest an $\mathrm{M}_{\mathrm{bol}} \sim-8$ to $-10 \mathrm{mag}$ $\left(\log L / L_{\odot}=5.5 \pm 0.4\right)$. Based on the IR O I triplet strength $(\mathrm{EW}=2.8 \AA)$, an $\mathrm{M}_{\mathrm{bol}}=-9.5 \mathrm{mag}\left(\log L / L_{\odot}=5.7\right)$ was estimated. A correction for the temperature dependence of the $\mathrm{EW}(\mathrm{OI})$ gives $\mathrm{M}_{\mathrm{bol}}=-9.1 \mathrm{mag}\left(\log L / L_{\odot}=5.5\right)$. However, taking into account the uncertainties, the most probable value is $\mathrm{M}_{\mathrm{bol}}=-9.5 \pm 0.4 \mathrm{mag}\left(\log L / L_{\odot}=5.7 \pm 0.2\right)$. The $\mathrm{H} \alpha$ and $\mathrm{H} \beta$ lines have double-peaked profiles with variable intensities. A weak He I line at $\lambda 5876 \AA$ is detected in all the spectra. In conclusion, IRC+10420 quickly evolves toward the WR phase. The presence of the Her $5876 \AA$ line at a so low temperature may be due to the high luminosity and/or an enhanced He abundance.

Acknowledgements. This research was supported in part by the U.S. Civilian Research \& Development Foundation, grant RP1-2264. We thank H. Levato, M. Grosso, R. J. Rudy, D. K. Lynch, S. Mazuk, R. Puetter, R. O. Gray, 
J. C. Wislon, P. Garica-Lario, J. Perea Calderon, N. Manset, T. Lloyd Evans, F. Marang, V. E. Panchuk, S. V. Ermakov, M. V. Yushkin, O. Ezhkova, K. S. Kuratov, T. A. Sheikina, A. V. Kusakin, and D. B. Mukanov for the observations they obtained for this project.

\section{References}

Allen, D. A., \& Swings, J.-P. 1976, A\&A, 47, 293

Carlson, E. D., \& Henize, K. G. 1979, Vistas Ast., 23, 213

Dong, Y. S., \& Hu, J. Y. 1991, Chin. Astr. Astrophys., 15, 275

Gledhill, T. M., et al. 2001, MNRAS, 322, 321

Klochkova, V. G., Panchuk, V. E., \& Tavolzhanskaya, N. S. 2002, Ast. Lett., 28,49

Klochkova, V. G., et al. 2002, Ast. Rep., 46, 139

Lamers, H. J. G. L. M., et al. 1998, A\&A, 340, 117

Lopes, D. F., Damineli Neto, A., \& de Freitas Pacheco, J. A. 1992, A\&A, 261, 482

Likkel, L., et al. 1991, A\&A, 246, 153

Miroshnichenko, A. S., et al. 2000, A\&AS, 144, 5

Miroshnichenko, A. S., et al. 2001, A\&A, 371, 600

Miroshnichenko, A. S., et al. 2002, A\&A, 383, 171

Neckel, Th., \& Klare, G. 1980, A\&AS, 42, 251

Oudmaijer, R. D. 1998, A\&AS, 129, 541

Reig, P., Fabregat, J., \& Coe, M. J. 1997, A\&A, 322, 193

Sheikina, T. A., Miroshnichenko, A. S., \& Corporon, P. 2000, In ASP Conf. Ser. Vol. 214, The Be-Phenomenon in Early-Type Stars, ed. M. A. Smith, H.

F. Hendrichs, \& J. Fabregat (San Francisco: ASP), 494

Voors, R. H. M. 1999, Ph. D. Thesis, Universiteit Utrecht, The Netherlands

Wolf, B., \& Stahl, O. 1985, A\&A, 148, 412

Zickgraf, F.-J. 2001, A\&A, 375, 122 